FTUV 97-50; IFIC 97-53

September 26, 2018

\title{
Towards an unified picture of constituent and current quarks $\dagger$
}

\author{
Sergio Scopetta and Vicente Vento ${ }^{(a)}$ \\ Departament de Fisica Tè̀rica, Universitat de València \\ 46100 Burjassot (València), Spain \\ and \\ (a) Institut de Física Corpuscular, Consejo Superior de Investigaciones Científicas \\ and \\ Marco Traini \\ Dipartimento di Fisica, Università di Trento, I-38050 Povo (Trento), Italy \\ and Istituto Nazionale di Fisica Nucleare, Gruppo Collegato di Trento
}

\begin{abstract}
Using a simple picture of the constituent quark as a composite system of point-like partons, we construct the parton distributions by a convolution between constituent quark momentum distributions and constituent quark structure functions. We evaluate the latter at a low hadronic scale with updated phenomenological information, and we build the momentum distributions using well-known quark models. The resulting parton distributions and structure functions are evolved to the experimental scale and good agreement with the available DIS data is achieved. When compared with a similar calculation using non-composite constituent quarks, the accord with experiment of the present calculation becomes impressive. We therefore conclude that DIS data are consistent with a low energy scenario dominated by composite, mainly non-relativistic constituents of the nucleon.
\end{abstract}

Pacs: 12.39-x, 13.60.Hb, 14.65-q, 14.70Dj

Keywords: hadrons, quarks, gluons, evolution, parton distributions, structure functions.

scopetta@titan.ific.uv.es

vicente.vento@uv.es

traini@science.unitn.it

$\dagger$ Supported in part by DGICYT-PB94-0080, DGICYT-PB95-0134 and TMR programme of the European Commision ERB FMRX-CT96-008. 


\section{Introduction}

The theory of hadronic interactions Quantum Chromodynamics, $(Q C D)$ [1], is a theory of quarks (antiquarks) and gluons, as has been shown in the asymptotic regime, where the interaction can be treated perturbatively [2]. At low energies, the idea that baryons are made up of three constituent quarks and mesons of a (constituent) quark-antiquark pair [3], the naive quark model scenario, accounts for a large number of experimental facts 《4. The quest for a relation between the two regimes, i.e., between the current quarks of the theory and the constituent quarks of the model has an old history [5] and, in recent years, this search has been the leitmotiv of a considerable research effort [6]. The fundamental problem one would like to understand is how confinement, i.e., the apparent absence of color charges and dynamics in hadron physics, is realized.

Detailed quark models of hadron structure based on the constituent quark concept have been defined in order to explain low energy properties [7, 8]. To proceed from these models to the asymptotic regime, where deep inelastic scattering (DIS) takes place, a hadronic scale is associated to the model calculations. The experimental conditions are reached by projecting the leading twist component of the observable and evolving according to perturbative $Q C D$ [9, 10]. The procedure describes succesfully the gross features of the DIS results [11]. In order to produce a more quantitative fit different mechanisms have been proposed: valence gluons, sea quarks and antiquarks, relativistic kinematics, etc... We will show that some of these mechanisms appear naturally if we endow the constituent quarks with structure, using a procedure already advanced in ref. [11].

It was long ago, at the time that $Q C D$ was being proposed, that a procedure, hereafter called ACMP [12], was proposed to understand the relation between the constituent quarks and the partons [13]. In this approach, which we here explore, constituent quarks are complex objects, made up of point-like partons (current quarks (antiquarks) and gluons), interacting by a residual interaction described by a quark model. The hadron structure functions are obtained as a convolution of the constituent quark wave function with the constituent quark structure function. This procedure has been recently revived to estimate the structure function of the pion with success 14.

In the ACMP approach, each constituent quark is dressed by a neutral cloud of quarkantiquark pairs and gluons, thus, this scenario supports a confinement mechanism a la De Rújula, Georgi and Glashow [7]. A few years earlier a second approach had been developed [15], in which the proton is assumed to be made out of three valence quarks plus a neutral core of quark-antiquark pairs and gluons, very much in the spirit of recent developments along the Manohar-Georgi model [16]. This duality of approaches has to do, in modern language, with the implementation of Chiral Symmetry Breaking (CSB). The naive models [7] do not contain spontaneous CSB, and this phenomenon has to be implemented if they are to represent $Q C D$ at low energies. But does it have to be done at the level of elementarity that Kuti and Weisskopf 15 proposed and the Manohar-Georgi [16] philosophy implies?

Summarizing: the ACMP scheme leads to parton distributions given by a convolu- 
tion between constituent quark momentum distributions and constituent quark structure functions; we will evaluate the latter at the low energy hadronic scale with updated phenomenological information and the former using well-known non-relativistic quark models of hadron structure [8, 17]; we will evolve the resulting parton distributions and structure functions to the experimental scale, to check if good agreement with the available DIS data is found.

\section{The theoretical framework}

In the picture we next explore [12], constituent quarks are themselves complex objects whose structure functions are described by a set of functions $\phi_{a b}(x)$ that specify the number of point-like partons of type $b$, which are present in the constituent of type $a$ with fraction $x$ of its total momentum. We will hereafter call these functions generically the structure functions of the constituent quarks.

The functions describing the nucleon parton distributions are expressed in terms of the independent $\phi_{a b}(x)$ and of the constituent probability distributions $u_{0}$ and $d_{0}$ as,

$$
f\left(x, Q^{2}\right)=\int_{x}^{1} \frac{d z}{z}\left[u_{0}\left(z, Q^{2}\right) \phi_{u f}\left(\frac{x}{z}, Q^{2}\right)+d_{0}\left(z, Q^{2}\right) \phi_{d f}\left(\frac{x}{z}, Q^{2}\right)\right]
$$

where $f$ labels the various partons,i.e., valence quarks $\left(u_{v}, d_{v}\right)$, sea quarks $\left(u_{s}, d_{s}, s\right)$, sea antiquarks $(\bar{u}, \bar{d}, \bar{s})$ and gluons $g$.

The different types and functional forms of the structure functions of the constituent quarks are derived from three very natural assumptions [12]:

i) The point-like partons are determined by $Q C D$, therefore, quarks, antiquarks and gluons;

ii) Regge behavior for $x \rightarrow 0$ and duality ideas;

iii) invariance under charge conjugation and isospin.

These considerations define in the case of the valence quarks the following structure function,

$$
\phi_{q q_{v}}\left(\frac{x}{z}, Q^{2}\right)=\frac{\Gamma\left(A+\frac{1}{2}\right)}{\Gamma\left(\frac{1}{2}\right) \Gamma(A)} \frac{(1-x)^{A-1}}{\sqrt{x}} .
$$

For the sea quarks the corresponding structure function becomes,

$$
\phi_{q q_{s}}\left(\frac{x}{z}, Q^{2}\right)=\frac{C}{x}(1-x)^{D-1},
$$

and in the case of the gluons we take

$$
\phi_{q g}\left(\frac{x}{z}, Q^{2}\right)=\frac{G}{x}(1-x)^{B-1} .
$$


The other ingredients of the formalism, i.e., the probability distributions for each constituent quark, are defined according to the procedure of Traini et al. [11], that is, a constituent quark, $q_{0}$, has a probability distribution determined by

$$
x q_{0}\left(z, \mu_{0}^{2}\right)=\frac{m_{q}}{M} \int d^{3} p n_{q}(\vec{p}) \delta\left(z-\frac{p^{+}}{M}\right),
$$

where $n_{q}(\vec{p})$ is its momentum distribution in the corresponding baryonic state. This very intuitive expression requires of a support correction [11].

Our last assumption relates to the scale at which the constituent quark structure is defined. We choose for it the so called hadronic scale $\mu_{0}^{2}$ [11, 19]. This hypothesis fixes all the parameters of the approach (Eqs. (22) through (14)). The constants $A, B, G$ and the ratio $C / D$ are determined by the amount of momentum carried by the different partons. We choose, $53.5 \%$ by the valence quarks and $35.7 \%$ by the gluons, which corresponds to a hadronic scale of $\mu_{0}^{2}=0.34 \mathrm{GeV}^{2}$ in agreement with the parametrization of [19]. $C$ (or $D$ ) is fixed according to the value of $F_{2}$ at $x=0$ [12], and its value is chosen again according to [19]. We stress that all these inputs are forced only by the updated phenomenology. The values of the parameters obtained are: $A=0.435, B=0.378, C=0.05, D=2.778$ and $G=0.135$. We, here, note that the unpolarized structure function $F_{2}$ is rather insensitive to the change of the sea $(C, D)$ and gluon $(B, G)$ parameters.

To complete the process [9, 10] the above input distributions are NLO-evolved in the DIS scheme to $10 \mathrm{GeV}^{2}$, where they are compared with the data.

\section{Results}

We will discuss the results for the proton in two models for $u_{0}$ and $d_{0}$ :

i) The non relativistic model of Isgur and Karl [8];

ii) The algebraic model of Bijker, Iachello and Leviatan [17].

The parameters of the models are kept as determined by their authors, which fitted them to static properties of hadrons.

In Figs. 1 through 5 we show the results of the present calculation. Figs. 1 and 2 contain the $u$ valence quark distribution, while Figs. 3 and 4 the $F_{2}$ structure function. We compare the results of the present calculation with those of the same calculation without considering the constituent quark structure. We stress that no fitting of the parameters to approach the unpolarized data has been done. The new parameters, introduced in the definition of the $\phi$ functions, have been solely determined by the definition of the hadronic scale and by the assumption of Regge behavior at low $x$, whose validity at low $Q^{2}$, has been recently confirmed [20]. The procedure has provided us automatically with the momentum sum rule and no ad hoc modifications of the model wave functions have been necessary. In Fig. 5 we show the gluon distribution, $x g\left(x, Q^{2}\right)$, at the experimental 
scale. The impossibility to determine this quantity in the extreme quark model scenario motivated the introduction of primordial gluons at the hadronic scale [11, 19].

The improvement of all of the results with respect to previous calculations is impressive. One should stress, that the momentum distribution of the model in ref. [17], understood as a constituent quark momentum distribution, leads to an amazing agreement with the data.

\section{Conclusions}

In previous work we found the limitations associated with naive quark model calculations when applied to the explanation of DIS data [11]. In that reference we analyzed several paths to extend the formalism to incorporate the underlying partonic structure in a natural way. A very appealing scheme seemed to us incorporating the assumption that constituent quarks are not elementary [12]. We have here explored this scenario. Partons (the quarks, antiquarks and gluons of $Q C D$ ) at the hadronic scale are generated by unveiling the structure of the constituent quarks. We have seen that incorporating this structure in a very physical way improves notably the agreement with the DIS data. From the point of view of the calculation, we must stress, that no parameters of the model have been changed with respect to the original fit to the low energy properties. The new parameters arising from the description of the constituent quark structure functions have been adjusted to describe the input scenario according to the hadronic scale philosophy. In this way the sea and gluon distributions are generated in a consistent way.

Taking into account the almost inexistent fit of parameters, the results are surprisingly good for both models [8, 17]. In particular the momentum distribution of ref. [17] seems to have been defined to fit the DIS data, which is not the case.

A quantitative comparison with the data, indicates that our calculation fails to reproduce the high $x$ tail of the experimental curves. Our previous experience tells us that a probable cause for this failure might be the lack of high-momentum components in the model wave function,i.e., the failure of the models to take into acount the relativistic motion of the constituent quarks, an omission which we hope soon to remedy [21].

The same analysis can be easily performed for the polarized case and it is in progress [21]. The idea being if this transformation to the partonic regime from the constituent regime is able to account for the so called spin crisis [22, 23, 24]. Moreover, since the method seems to be very predictive, we are confident that it could be useful to estimate unmeasured quantities, such as the transversity parton distribution $h_{1}$ [25], whose quark model analysis has been already addressed [26].

The introduction of composite quarks is the most natural way to solve the old Melosh problem and to understand some of the more recent ones [22, 23]. As many times in physics, the relation between constituent and current quarks, has not come from a symmetry transformation but from unveiling some underlying structure. Constituent and current quarks are not at the same level of elementarity. 
The problem of CSB has not been directly addressed in this paper. CSB must be implemented in any low energy model. If the implementation has to take place at the level of the composite constituent quarks or at the level of the current quarks is a subject of debate [27, 28]. Our results seem to imply that it is at the level of the constituent quarks that CSB has to be introduced, i.e., we retake the beautiful discussion in [12], and confer to Regge behavior a fundamental rôle, which has to be mantained by the chosen CSB mechanism.

We feel safe to conclude that, the current quarks seen at the parton level seem to be embedded in the composite constituent quarks seen at lower $Q^{2}$. An unified picture of current quarks, succesfully describing DIS, and constituent quarks, succesfully describing static properties, is possible. Work is in progress in that direction.

\section{Acknowledgements}

We are grateful to Franco Iachello for a clarifying correspondence regarding the model of Bijker, Iachello and Leviatan. This work was finished while one of the authors (VV) was visiting the Dipartimento di Fisica dell'Università di Trento. He acknowledges partial financial support from INFN and the warm hospitality of the members of the Dipartimento.

\section{References}

[1] H. Fritsch, M. Gell-Mann and H. Leutwyler, Phys. Lett. 47 B (1973) 365.

[2] F. Yndurain, Quantum Chromodynamics (Springer Verlag, Heidelberg 1983); T.Mutha, Foundations of Quantum Chromodynamics (World Scientific, Singapore 1987); R.G. Roberts, The structure of the proton (Cambridge University Press, Cambridge (U.K.) 1990); R.D. Field, Applications of perturbative QCD (Addison-Wesley, Reading, MA, 1989).

[3] M. Gell-Mann, Phys. Lett. 8 (1964) 214; G. Zweig, CERN reportTH401 (1964) 412, unpublished.

[4] J.J.J. Kokkedee, The Quark Model (W.A. Benjamin, New York,1969); D.B. Lichtenberg, Unitary Symmetry and Elementary Particles (Academic Press, New York, 1978); F.E. Close, An Introduction to Quarks and Partons (Academic Press, New York 1979).

[5] H.J. Melosh, Phys. Rev. D9 (1974) 1095.

[6] M. Traini, L. Conci and U. Moschella, Nucl. Phys. A 544 (1992) 731; F.M. Steffens and A.W. Thomas, Nucl. Phys. A 568 (1992) 798; C.J. Benesh, T. Goldman and G.J. Stephenson Jr., Phys. Rev. C 48 (1993) 1379; H.J. Weber, Phys. Rev. D 49 (1994) 3160 . 
[7] A. De Rújula, H. Georgi and S.L. Glashow, Phys. Rev. D 12 (1975) 147.

[8] N. Isgur and G. Karl, Phys. Rev. D 18 (1978) 4187, D 19 (1979) 2653, D 23 (1981) $817(\mathrm{E})$.

[9] G. Parisi and R. Petronzio, Phys. Lett. B 62 (1976) 331.

[10] R.L. Jaffe and G.G. Ross, Phys. Lett. B 93 (1980) 313.

[11] M. Traini, V. Vento, A. Mair and A. Zambarda, Nucl. Phys. A 614 (1997) 472.

[12] G. Altarelli, N. Cabibbo, L. Maiani and R. Petronzio Nucl. Phys. B 69 (1974) 531.

[13] R.P. Feynman, Photon-Hadron Interaction (W.A. Benjamin, New York 1972).

[14] G. Altarelli, S. Petrarca and F. Rapuano, Phys. Lett. B 373 (1996) 200.

[15] J. Kuti and V.F. Weisskopf, Phys. Rev. D 11 (1971) 3418.

[16] A.Manohar and H. Georgi, Nucl. Phys. B 234 (1984) 189.

[17] R. Bijker, F. Iachello and A. Leviatan, Ann. Phys. 236 (1994) 69; Phys Rev. C 54 (1996) 1935; Phys. Rev D 55 (1997) 2862.

[18] H. L. Lai et al., Phys. Rev. D 51 (1995) 4763.

[19] M. Glück, E. Reya and A. Vogt Z. Phys. C 53 (1992) 127.

[20] G. Altarelli, R.D. Ball, S. Forte and G. Ridolfi, hep-ph/9701289, Nucl. Phys. B to appear.

[21] S. Scopetta, V. Vento and M. Traini, in preparation.

[22] R.L. Jaffe and A.V. Manohar, Nucl. Phys. B 337 (1990) 509.

[23] H. Fritzsch, Nucl. Phys. (Proc. Suppl.) B 23 (1991) 91.

[24] G. Altarelli and G. Ridolfi, Nucl. Phys. (Proc. Suppl.) B 39 (1995) 106.

[25] J. Ralston and D.E. Soper, Nucl. Phys. B 152 (1979) 1; R.L. Jaffe and X. Ji, Phys. Rev. Lett. 67 (1991) 552; Nucl. Phys. B 375 (1992) 527; R. Jaffe, Erice Lectures 1995.

[26] M. Stratmann, Z. Phys. C 60 (1993) 763; R.L. Jaffe and X. Ji, Phys. Rev. D 49 (1994) 114; R. Jacob, P.J. Mulders and J. Rodrigues, hep-ph/9704335; S. Scopetta and V. Vento, hep-ph/9706413.

[27] S.A. Kulagin, W. Melnitchouk, T. Weigl and W. Weise, Nucl. Phys. A 597 (1996) 515.

[28] R.D. Ball and S. Forte, Nucl. Phys. (Proc. Suppl.) B 39 (1995) 28. 


\section{Captions}

Figure 1: We show the parton distribution $x u_{V}\left(x, \mu_{0}^{2}\right)$ obtained at the hadronic scale $\mu_{0}^{2}=0.34 \mathrm{GeV}^{2}$ for a) the Isgur-Karl based model [8] with $36 \%$ valence gluons at the hadronic scale [11] (dot-dashed) and b) the present convolution approach based on the pure Isgur-Karl wave functions (long-dashed). The same distributions, $x u_{V}\left(x, Q^{2}\right)$, evolved at NLO and at a scale of $Q^{2}=10 \mathrm{GeV}^{2}$, are given by the dashed and full curves, respectively. The fit of the data $[18]$ at $Q^{2}=10 \mathrm{GeV}^{2}$ is also shown for comparison (dots).

Figure 2: Caption as in Figure 1 for the model of Bijker, Iachello and Leviatan [17. Again the corresponding valence gluon model is that of ref.[1] and the data those of ref. 18.

Figure 3: We show the structure function $F_{2}\left(x, Q^{2}\right)$ obtained by NLO-evolution to $Q^{2}=10 \mathrm{GeV}^{2}$ in the present convolution approach, using the wave functions of [8] (full). The result of [11] (dashed) for the same quantity is shown. The data at $Q^{2}=10 \mathrm{GeV}^{2}$, corresponding to the analysis (without heavy quark sea) of Lai et al. [18], are plotted for comparison (dots).

Figure 4: Caption as in Figure 3 for the model of Bijker, Iachello and Leviatan [17]. Again the corresponding valence gluon model is that of ref. 11] and the data are those of ref. 18 .

Figure 5: We show the gluon distribution $x g\left(x, Q^{2}\right)$ at $Q^{2}=10 \mathrm{GeV}^{2}$ obtained with the present approach for the two models investigated: a) Isgur and Karl [8] (dot-dashed) ; b) Bijker, Iachello and Leviatan [17 (long-dashed). The data are those of ref. 18. 


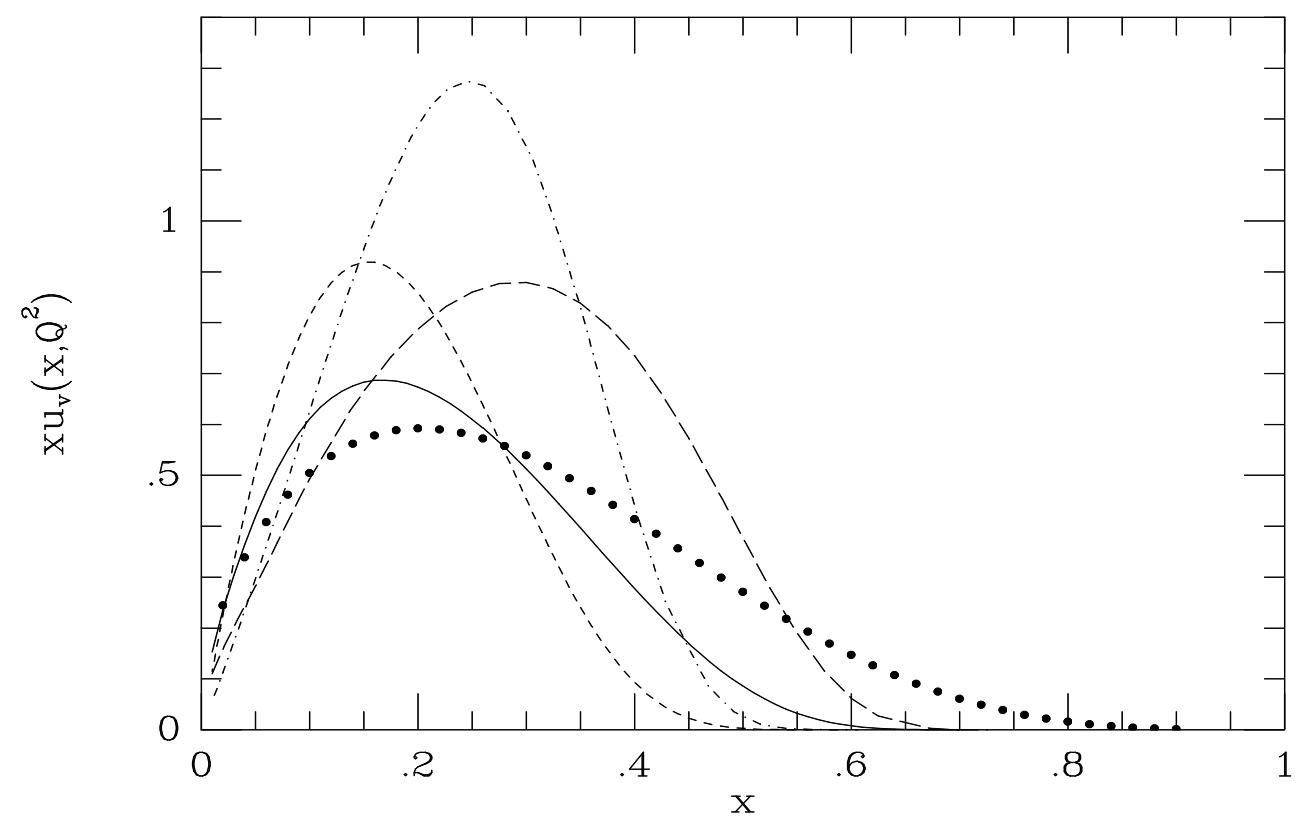

S. Scopetta, V. Vento and M. Traini

FIGURE 1 


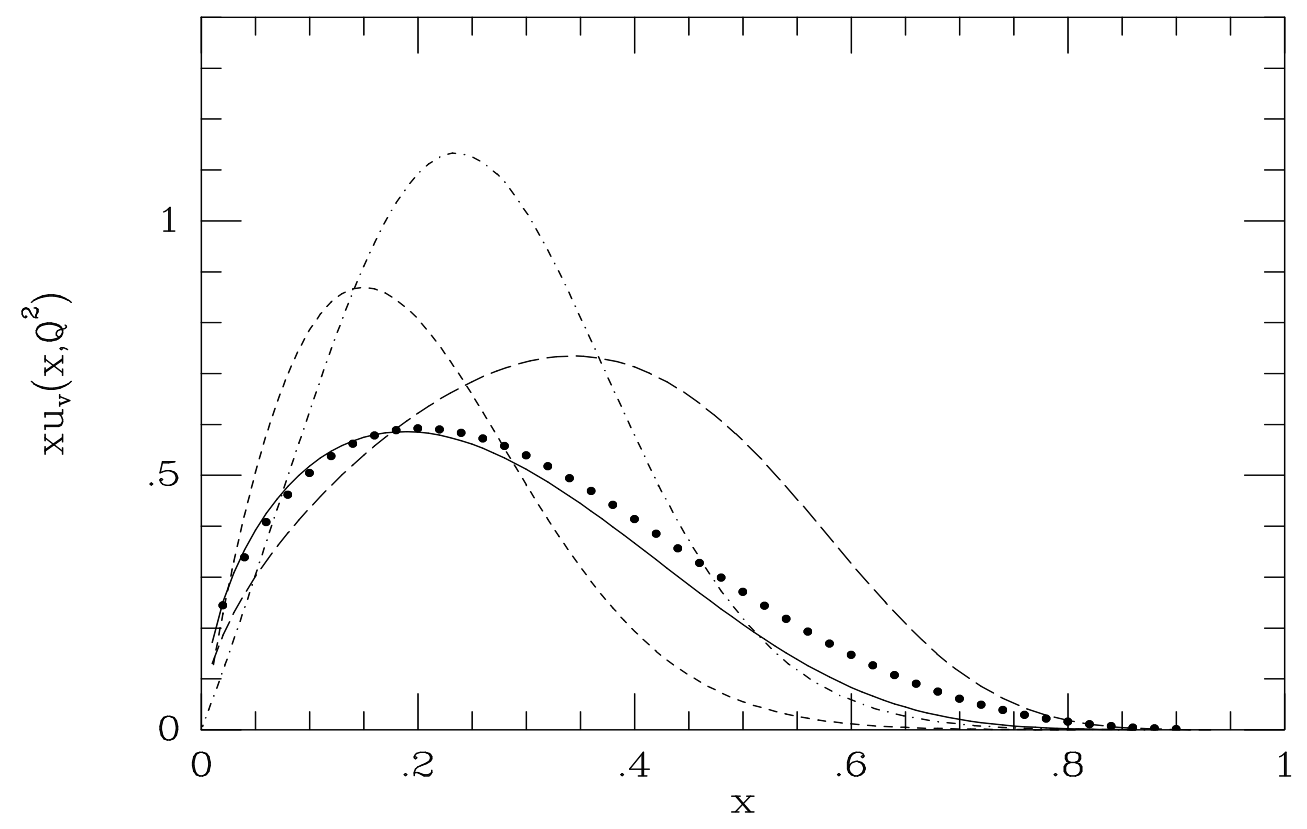

S. Scopetta, V. Vento and M. Traini

FIGURE 2 


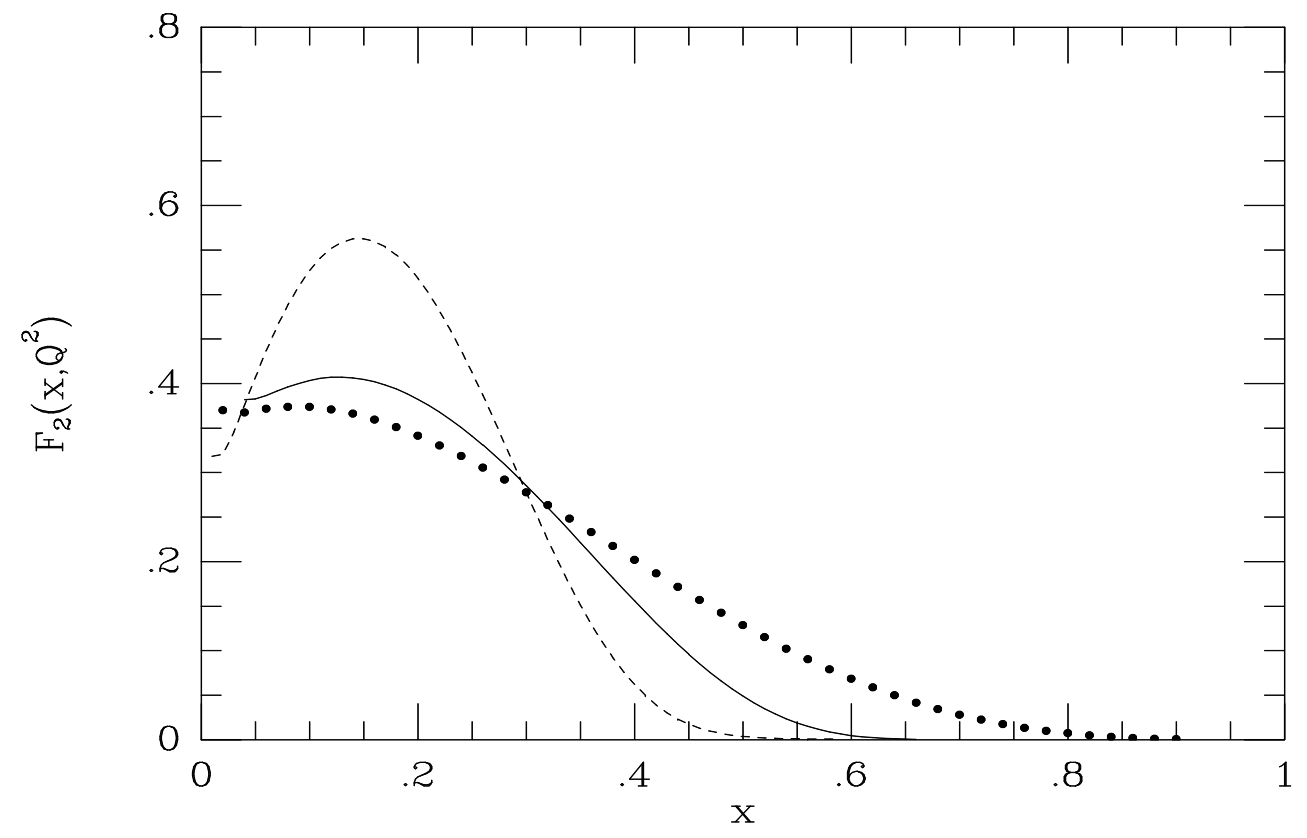

S. Scopetta, V. Vento and M. Traini

FIGURE 3 


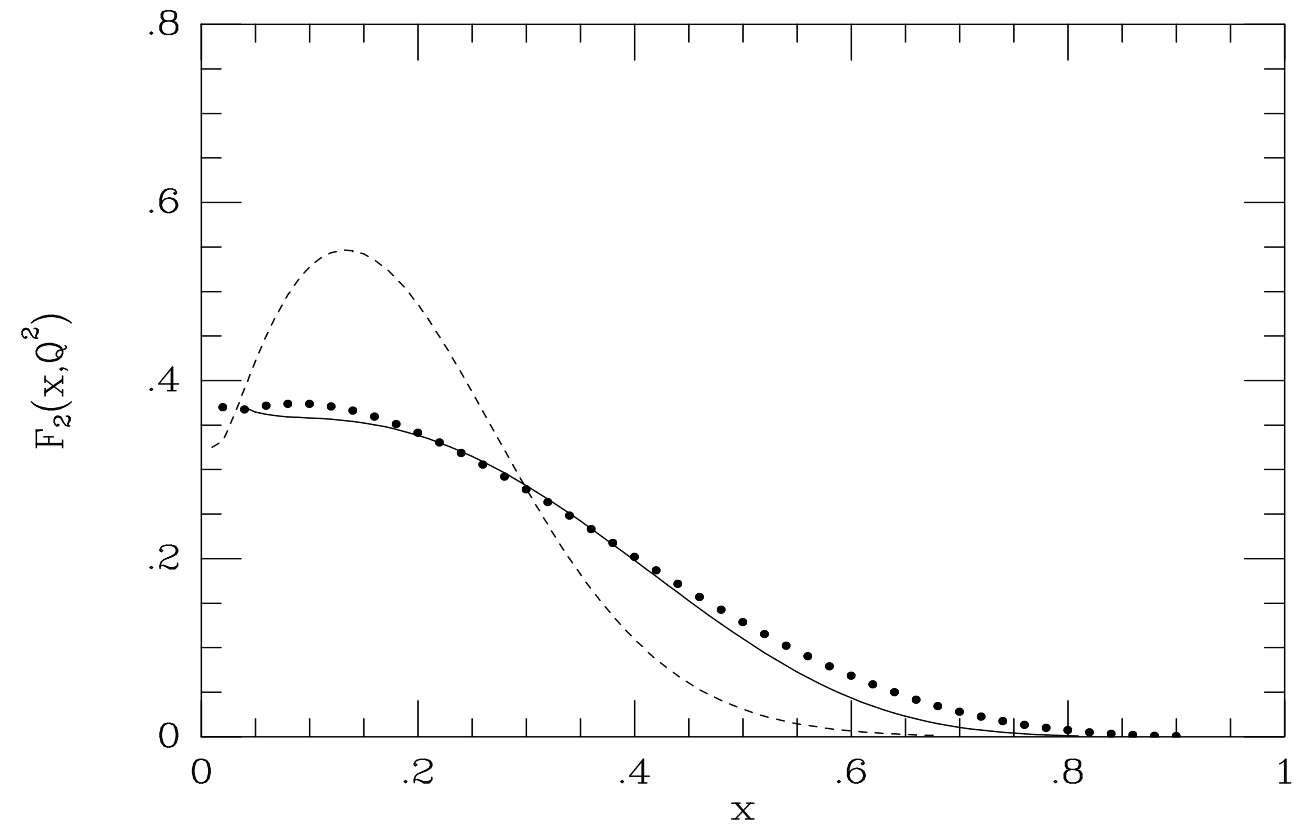

S. Scopetta, V. Vento and M. Traini

FIGURE 4 


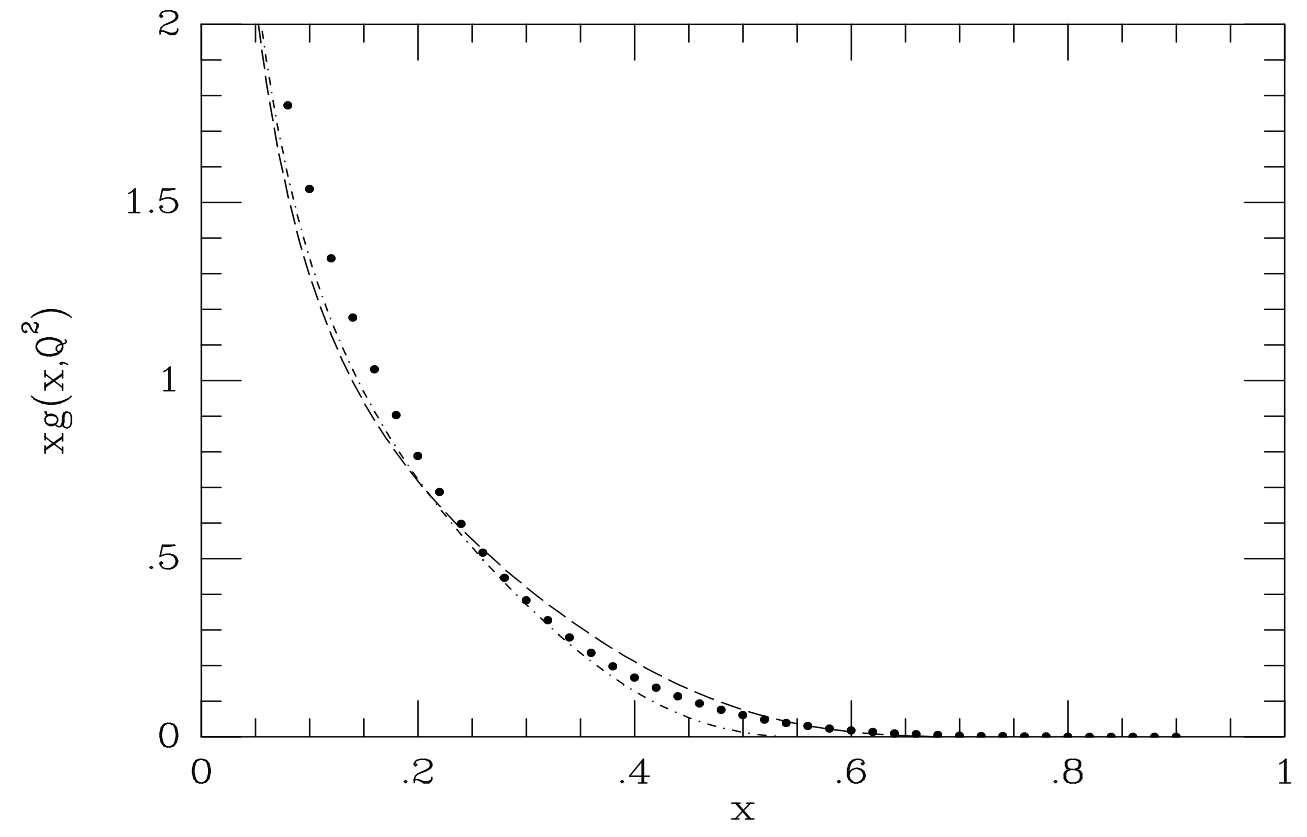

S. Scopetta, V. Vento and M. Traini

FIGURE 5 